Aus Abb. 8 ist folgendes ersichtlich. Untersuchen wir die Existenz des Feldbogens mit $q=10$, so wären nach der reinen Feldtheorie wie auch nach der $\mathrm{T}-\mathrm{F}$-Theorie Stromdichten von $3-4 \cdot 10^{7} \mathrm{~A} / \mathrm{cm}^{2}$ erforderlich. Bei Berücksichtigung der individuellen Feldkomponente kann dagegen der Bogen in Übereinstimmung mit dem experimentellen Befund schon mit $2 \cdot 10^{6} \mathrm{~A} / \mathrm{cm}^{2}$ existieren. Mit zunehmendem $q$ nimmt der Einfluß der individuellen Feldkomponente scheinbar ab. Hierzu ist jedoch zu bemerken, daß die Festsetzung $F_{\mathrm{e}}=F_{\mathrm{n}}$ nur eine untere Grenze des Effektes liefert. Dies macht sich gerade bei den großen $q$-Werten bemerkbar.
Wir fassen zusammen:

Ohne Berücksichtigung der individuellen Feldkomponente erstreckt sich der Existenzbereich des Feldbogens nur dann bis zu den gemessenen Stromdichten von einigen $10^{6} \mathrm{~A} / \mathrm{cm}^{2}$ herunter, wenn wir extreme Temperatur der Kathode, Erniedrigung des Austrittspotentials oder einen field-enhancementfactor annehmen ${ }^{9}$. Berücksichtigen wir dagegen den Einfluß der individuellen Feldkomponente, so wird der Existenzbereich erheblich nach niedrigeren Stromdichten verschoben. In diesem Falle können wir die gemessenen Stromdichten bereits ohne die Zusatzannahme erklären.

\title{
Messungen der Driftbeweglichkeit, des elektrischen Widerstandes und der Lebensdauer von Minoritätsladungsträgern an $n$ - und p-Germanium bei hohen hydrostatischen Drucken*
}

\author{
Von Gottrried Landwehr ** \\ (Mitteilung aus der Physikalisch-Technischen Bundesanstalt) \\ (Z. Naturforschg. 14 a, 520-531 [1959]; eingegangen am 28. Februar 1959)
}

\begin{abstract}
Die Driftbeweglichkeit von Löchern und Elektronen in Germanium wurde bei Drucken bis zu $14000 \mathrm{kp} / \mathrm{cm}^{2}$ mit einem Brücken-Impuls-Verfahren gemessen, das auf der Leitfähigkeitsmodulation durch injizierte Ladungsträger beruht. Die Löcherdriftbeweglichkeit stieg linear mit dem Druck um $(1,39 \pm 0,27) \%$ bei $10000 \mathrm{kp} / \mathrm{cm}^{2}$. Die Elektronendriftbeweglichkeit nahm mit steigendem Druck stärker als proportional ab, um $8 \%$ bei $10000 \mathrm{kp} / \mathrm{cm}^{2}$ und um $15 \%$ bei $14000 \mathrm{kp} / \mathrm{cm}^{2}$. Die Zunahme der Löcherdriftbeweglichkeit kann bei Berücksichtigung der Druckabhängigkeit der elastischen Konstanten durch ein Anwachsen der effektiven Masse der schweren Löcher um $1 \%$ bei $10000 \mathrm{kp} / \mathrm{cm}^{2}$ gedeutet werden. Bei den Elektronen kommt zur Beweglichkeitsänderung auf Grund von Änderungen der effektiven Masse und der elastischen Eigenschaften des Germaniums ein Anteil, der durch eine Umordnung im E-Raum hervorgerufen wird. Die Ursache für die rasche Beweglichkeitsabnahme bei höheren Drucken ist in der Besetzung des [100]-Minimums im f-Raum auf Kosten des [111]-Minimums zu suchen, die Anlaß zu einer Streuung der Elektronen zwischen den beiden Extrema gibt. Die Änderungen der Driftbeweglichkeit stimmen innerhalb der Meßgenauigkeit mit den aus Widerstands. messungen an n- und p-Germanium abgeleiteten Änderungen der Leitfähigkeitsbeweglichkeit überein. Daraus kann geschlossen werden, daß sich der Ionisationszustand von Donatoren und Akzeptoren durch Anwendung hoher Drucke nicht ändert und daß keine Haftstellen auftreten. Aus der Differenz der prozentualen Widerstandsänderungen eines $5 \Omega \mathrm{cm}$ - und eines $11 \Omega \mathrm{cm}$-Ge-Kristalles, die gleichzeitig dem Druck ausgesetzt wurden, konnte der Druckkoeffizient der verbotenen Zone zu $4,9 \cdot 10^{-6}$ $\mathrm{eV} \mathrm{cm} / \mathrm{kp}$ bestimmt werden, sowie die Eigenleitungskonzentration im Germanium bei $19,5^{\circ} \mathrm{C} \mathrm{zu}$ $2,41 \cdot 10^{26} / \mathrm{cm}^{3}$. An mehreren Kristallen wurde mit einem Brücken-Doppelimpulsverfahren die Minoritätsladungsträger-Lebensdauer unter Druck gemessen. Mit wachsender Zahl der Druckanwendungen fiel die effektive Lebensdauer auf etwa ${ }^{1 / 3}$ des Anfangswertes und war schließlich nur noch schwach druckabhängig.
\end{abstract}

Durch Anwendung hoher Drucke ist man imstande, die Gitterkonstante von Festkörpern zu verändern und damit deren elektrische Eigenschaften. Das Ziel, über eine rein phänomenologische Beschreibung der Druckeffekte hinauszukommen, dürfte beim Germanium, dem wohl am besten untersuchten Festkörper,

* Teilweise vorgetragen auf der Tagung des Verbandes Deutscher Physikalischer Gesellschaften, Heidelberg 1957, s. Phys. Verh. 8, 167 [1957].

** Braunschweig. am ehesten zu erreichen sein. Die Energiebänderstruktur von n- und p-Germanium ist weitgehend geklärt, für die elektrischen Eigenschaften, insbesondere die Ladungsträgerbeweglichkeit, liegt eine detaillierte Theorie vor. Es besteht somit Aussicht, daß die durch Anwendung hoher hydrostatischer Drucke hervorgerufenen Änderungen der Elektronen- und Löcherbeweglichkeit gedeutet werden können, d. h. zurückgeführt auf Änderungen der effektiven Masse, der elastischen Konstanten und ganz allgemein der 
Energiebänderstruktur. Um Aufschluß über das Beweglichkeitsverhalten von Ladungsträgern unter Druck zu erhalten, kann man die spezifische Leitfähigkeit von n- und p-Material im Erschöpfungsbereich messen ${ }^{1}$; denn bei konstanter Ladungsträgerdichte ist die spezifische Leitfähigkeit der Leitfähigkeitsbeweglichkeit proportional, die bei nicht zu stark dotierten Kristallen mit der Driftbeweglichkeit übereinstimmt. Da aber die Annahme, daß die Trägerzahl bei Druckanwendung unverändert bleibt, nicht a priori gesichert ist, verdient eine direkte Beweglichkeitsmessung den Vorzug. Aus diesem Grunde wurde die Druckabhängigkeit der Driftbeweglichkeit von Minoritätsladungsträgern in $n$ - und p-Germanium untersucht und die Ergebnisse der Fundamentalmessung mit den indirekt erzielten Resultaten verglichen. Die Leitfähigkeitsmessungen unter Druck lieferten gleichzeitig den Druckkoeffizienten der verbotenen Zone und die Eigenleitungskonzentration in Germanium bei Zimmertemperatur. Da sich im Laufe der Untersuchungen ergab, daß die Lebensdauer von injizierten Ladungsträgern vom Druck abhing, wurden an einigen Kristallen Lebensdauermessungen vorgenommen.

\section{Druckapparatur}

Der allseitige Druck wurde mit einer Hochdruckpresse nach J. BASSET durch Kompression einer Flüssigkeit erzeugt. Der Kopf der Apparatur ist schematisch in Abb. 1 dargestellt. Auf einen Zylinder aus Stahl hoher Festig. keit und Dehnung, der zwei zueinander senkrechte Boh. rungen von $19 \mathrm{~mm} \phi$ aufweist, ist ein zweiter Stahl. zylinder aufgeschrumpft. Der innere Zylinder erhält dadurch eine Vorspannung, die der Beanspruchung durch Innendruck entgegengerichtet ist. Eine Erhöhung des maximal herstellbaren Drucks wurde durch Autofrettage der Hochdruckkammer erreicht, d. h. die inneren Zonen des Innenzylinders wurden durch hohen Druck zum plastischen Fließen gebracht. Mit der Apparatur lassen sich Drucke bis $17000 \mathrm{kp} / \mathrm{cm}^{2}$ erzielen; wenn man eine geringere Lebensdauer in Kauf nimmt, auch Drucke bis $20000 \mathrm{kp} / \mathrm{cm}^{2}$. Der Druck erzeugende Stempel wird durch eine hydraulische Niederdruckpresse in die Zylinderbohrung getrieben. In den Kopf der Presse sind Kühlschlangen eingefräst, an die ein Thermostat angeschlossen wurde. Es wurde stets darauf geachtet, daß sich vor jeder Ablesung das Temperaturgleichgewicht eingestellt hatte. Nach jeder Kompression oder Expan-

1 P.W. Bridgman, Proc. Amer. Acad. Arts. Sci. 82, 17 [1953].

2 R. Lawrence u. A. F. Gibson, Proc. Phys. Soc., Lond. B 65, 994 [1952].

3 J. R. Haynes u. W. Shockley, Phys. Rev. 81, 835 [1951].

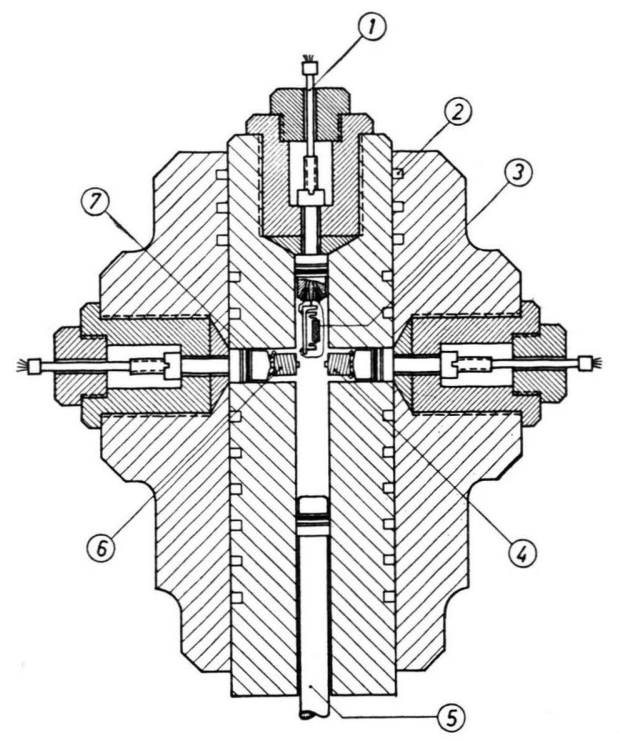

Abb. 1. Kopf der Hochdruckapparatur, schematisch. 1 Elektrische Dreifachdurchführung, 2 Kühlschlangen, 3 Germaniumkristall, 4 Manganinmanometer, 5 Kolben, 6 Platin-Widerstandsthermometer, 7 Bridgeman-Dichtung.

sion wurde mindestens 20 Min. gewartet, um die $\mathrm{Ab}$ weichung der Temperatur vom Sollwert kleiner als $1 / 10$ Grad zu halten, was durch ein eingebautes Platinwiderstandsthermometer kontrolliert wurde. Zur Druckmessung diente ein Manganinwiderstandsmanometer, das mit Hilfe einer Druckwaage kalibriert war. Der Widerstand der Manganinspule wurde mittels Normalvergleichswiderstand und einem Kompensator nach DiesseLHORST gemessen, auch die Temperaturmessung erfolgte auf diese Weise. Der Widerstand der Germaniumkristalle wurde mit einer Präzisions-WHeatstone-Brücke ermittelt. Germaniumkristall, Widerstandsmanometer und Platinthermometer wurden mittels hochisolierender elektrisch abgeschirmter Dreifachdurchführungen in die Druckkammer eingeführt. Die Isolation der Hochdruckdurchführungen, die bei Drucken bis zu $14000 \mathrm{kp} / \mathrm{cm}^{2}$ erprobt wurden, bestand aus dem Werkstoff Novotext. Als druckübertragende Flüssigkeit diente ein Gemisch der Zusammensetzung 50\% Petroleum, 25\% Paraffinöl, $25 \%$ Dekalin.

\section{Driftbeweglichkeits- und Lebensdauer- Meßmethode}

Da Druckeffekte in der Regel nicht groß sind, wurde von vornherein eine möglichst hohe Genauigkeit bei den Driftbeweglichkeitsmessungen angestrebt. Zu diesem Zweck wurde die Methode von Lawrence und Gibson ${ }^{2}$, die im Prinzip auf Haynes und SHockley ${ }^{3}$ zurückgeht, etwas abgeändert (Abb. 2). Bei der gewählten Schaltung sperrt der Rechteck- 


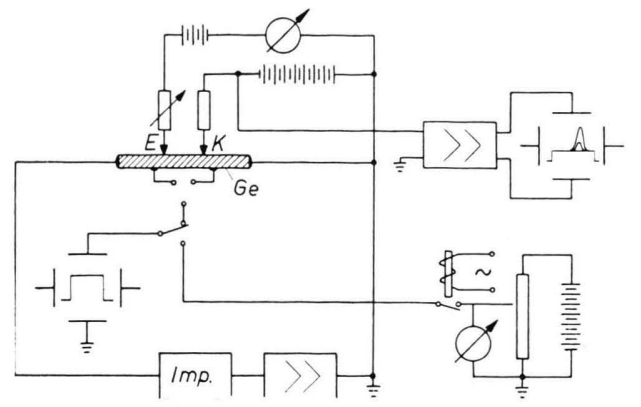

Abb. 2. Schaltung zur Messung der Löcherdriftbeweglichkeit. Ge Germaniumkristall, E Emitter, K Kollektor, Imp. Rechteckimpulsgenerator.

impuls des Absaugfeldes den Emitterstrom und es entsteht ein "Löcherpaket“. Auf diese Weise ist es möglich, die Impulslaufzeit bei verschiedenen Emitterstromstärken zu messen und auf die Stromstärke Null zu extrapolieren. Dadurch werden alle durch Leitfähigkeitsmodulation der injizierten Träger hervorgerufenen Effekte eliminiert ${ }^{4}$. Die Wahl von nicht zu kleinen Emitterströmen in Verbindung mit hinreichend hohen Absaugfeldstärken liefert schmale Löcherimpulse und gestattete eine recht genaue Bestimmung der Impulsspitzen. Die Messung der Impulslaufzeit, d. h. derjenigen Zeit, die vom Einsetzen des Rechteckimpulses bis zu dem Zeitpunkt vergeht, an dem der Löcherstrom sein Maximum erreicht hat, ist durch Überlagerung von Zeitmarken auf dem Oszillogramm möglich. Die Zeitmessung läßt sich aber mit höherer Genauigkeit durchführen: Man stellt die Spitze des Löcherimpulses auf die Mitte des Fadenkreuzes vor dem Oszillographenschirm ein, bei ausreichender Dehnung der Zeitachse. Die Dauer des Rechteckimpulses wird solange reduziert, bis er gerade dann aussetzt, wenn der Löcherimpuls sein Maximum erreicht hat. Die zugehörige Zeit wird auf der Skala des Impulsgenerators abgelesen. Die Messung der Feldstärke erfolgte in üblicher Weise mittels eines zweiten Kathodenstrahloszillographen durch Vergleich mit einer definierten Rechteckgleichspanung. Auf diese Weise wurde eine Reproduzierbarkeit, die mit der Meßgenauigkeit von Beweglichkeitsänderungen durch Druckeinfluß etwa zusammenfiel, von ca. $\pm 2 \%$ erreicht. Eine Herabsetzung der Meßunsicherheit war erstrebenswert, da es sich zeigte, daß die Löcherdriftbeweglichkeit sich nur schwach mit dem Drurck änderte und innerhalb der Fehlergrenzen keine sichere Entscheidung über die

4 W. v. Roosbroeck, Bell Syst. Techn. J. 29, 560 [1950].

5 A. Many, Proc. Phys. Soc., Lond. B 67, 9 [1954].
Druckabhängigkeit getroffen werden konnte. Außerdem wurde festgestellt, daß die Impulshöhe druckabhängig war. Dieser Effekt wurde als Änderung der Löcherlebensdauer gedeutet und ließ eine direkte Messung dieser Größe wünschenswert erscheinen. Da Emitter und Kollektor ihre Eigenschaften bei Druckanwendung ändern können, läßt sich die Methode unter den gegebenen Verhältnissen nicht für Lebensdauermessungen verwenden.. Aus diesem Grunde wurde die Driftbeweglichkeit von Löchern und Elektronen mit einem anderen Verfahren gemessen, das gleichzeitig Beweglichkeit und Lebensdauer zu ermitteln gestattet und überdies präziser ist.

Benutzt wurde die Brückenmethode von $\mathrm{M}_{\mathrm{ANY}}{ }^{5,6}$ in Verbindung mit einem Doppelimpulsgenerator (Abb. 3).

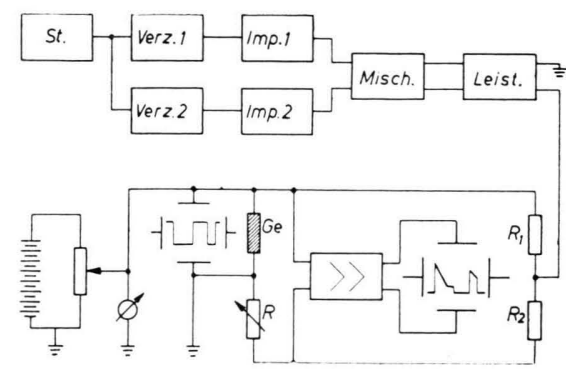

Abb. 3. Brückenschaltung zur Driftbeweglichkeits- und Lebensdauermessung. Ge Germaniumkristall, $R, R_{1}, R_{2}$ Widerstände, St Steuergenerator, Verz Verzögerungsstufe, Imp Rechteckimpulsgenerator, Misch Mischstufe, Leist Leistungsstufe.

Ein Einkristall aus Germanium, dessen Länge groß ist gegenüber den Querabmessungen, liegt in einer Widerstandsbrücke. Als Indikator dient ein Tektronix-Kathodenstrahloszillograph mit einem Breitband-Differentialverstärkereinsatz. Als Generator wird ein Doppelimpulsgenerator von WANDEL und Goltermans verwendet, der praktisch konstante Spannungen liefert und gestattet, die Dauer beider Rechteckimpulse unabhängig voneinander sowie deren zeitlichen Abstand zu variieren. Zur Erläuterung des Verfahrens diene die Messung der Elektronendriftbeweglichkeit in p-Germanium. Der zweite Impuls, der für die Beweglichkeitsmessung nicht gebraucht wird, sei zunächst abgeschaltet. Beim Einsetzen des ersten Impulses werden am negativ gepolten Ende des Kristalls, der mit einem schwach injizierenden Kontakt versehen ist, Elektronen injiziert. Diese werden vom elektrischen Feld erfaßt, durch den Kristall gesaugt und verursachen eine Leitfähigkeitszunahme. Die Absaug. feldstärke wird so hoch gewählt, daß die Lebensdauer der injizierten Elektronen groß ist gegenüber der Zeit, die zur Durchquerung des Kristalls benötigt wird. Dadurch ändert sich die Leitfähigkeit in erster Näherung linear mit der Zeit, und zwar so lange, bis die zuerst injizierten Elektronen das andere, mit einem möglichst

${ }^{6}$ R. Bray, Phys. Rev. 100, 1047 [1955]. 
sperrfreien Kontakt versehene Ende des Kristalls erreicht haben. Es stellt sich dann ein stationärer Zustand ein, auf dem Oszillogramm ist ein ziemlich scharfer Knick zu beobachten (Abb. 4, 1. Impuls), der es gestat-

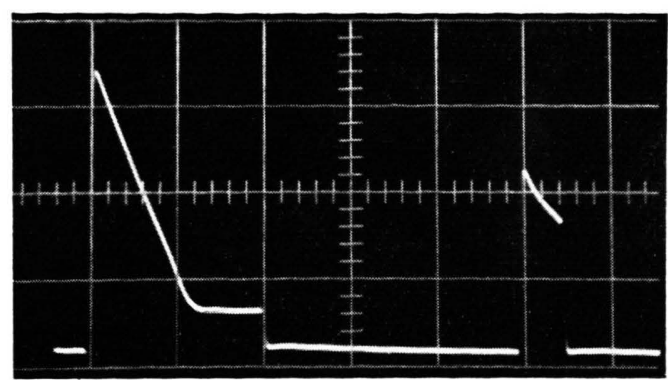

Abb. 4. Zeitlicher Verlauf des Widerstandes bei einem p-Germanium-Kristall in der Schaltung nach Abb. 3. Zeitmaßstab: $10 \mu$ s pro Masche des Gitters. Elektronenlebensdauer: $34 \mu \mathrm{s}$.

tet, die Elektronenlaufzeit zu messen. Mit einem zweiten Oszillographen und einer Vergleichs-Gleichspannung wird die am Kristall liegende Spannung gemessen, die zusammen mit Kristallänge und Laufzeit die Beweglichkeit $\mathrm{zu}$ berechnen gestattet. Auch bei den verwendeten schwach injizierenden Endkontakten, die Voraussetzung des Verfahrens sind und gestatten, Diffusionseffekte unberücksichtigt zu lassen, erwies es sich manchmal als notwendig, die Ladungsträgerlaufzeit bei mehreren, unterschiedlichen Injektionsverhältnissen $\mathrm{zu}$ messen und auf die Injektion Null zu extrapolieren. Der verwendete Oszillograph hatte eine lineare Zeitablenkung, die mittels Quarzuhrenfrequenzen kalibriert wurde. Die Laufzeitmessung geschah in der Weise, daß die Oszillogramme photographiert und anschließend projiziert und vermessen wurden. Bei je 5 Einzelmessungen der Feldstärke wurde eine Reproduzierbarkeit von mindestens $\pm 1 \%$ erzielt. Ein Vorteil gegenüber der Methode von Lawrence und Gibson liegt darin, daß nur 2 anstatt 4. Elektroden benötigt werden, daß Fehler durch die endliche Breite von Emitter und Kollektor nicht auftreten und daß die Bahnen der Ladungsträger stets parallel zum Feld laufen.

Die Brückenmethode wurde ursprünglich dazu benutzt, in Verbindung mit einem RC-Glied in einem Brückenzweig Lebensdauern zu messen. Dieses Verfahren läßt sich bei den verwendeten Endkontakten mit sehr niedrigem Injektionsverhältnis jedoch nicht anwenden wegen der Kopplung von angelegter Spannung und Injektionsverhältnis. So betrug z. B. das Injektionsverhältnis bei einer Feldstärke von $30 \mathrm{~V} / \mathrm{cm}$ etwa $1 \%$, bei $40 \mathrm{~V} / \mathrm{cm}$ etwa $3 \%$. Zweck der geringen Injektion war, Diffusionseffekte zu vermeiden und die Leitfähigkeitsmodulation klein zu halten. Bei den niedrigen Spannungen, die man an den Kristall legen müßte, um die Ladungsträgerlaufzeit groß gegenüber der Lebensdauer zu machen, wäre das Injek- tionsverhältnis so klein, daß eine extrem hohe Verstärkerempfindlichkeit erforderlich wäre. Aus diesem Grund wurde die Minoritätsladungsträger-Lebensdauer nach einem anderen Verfahren gemessen.

Nach dem Aussetzen des ersten Impulses in der Schaltung nach Abb. 3 rekombinieren die injizierten Elektronen mit Majoritätsladungsträgern. Die Zahl der Elektronen, welche zur Zeit $t$ nach dem Abschalten des ersten Impulses noch vorhanden sind, kann man dadurch ermitteln, daß man zu diesem Zeitpunkt einen zweiten Impuls konstanter Spannung an den Kristall anlegt. Die Höhe dieses Impulses ist ein Maß für die Lebensdauer. Wenn man die Verzögerungszeit $t$ kontinuierlich verändert, beschreibt die Spitze des zweiten Impulses eine Kurve, die den Verlauf der relativen Widerstandsänderung angibt und die sich (für Elektronen in p-Germanium) durch den Ausdruck

$$
\frac{\Delta R}{R_{0}}=\frac{n\left(t_{1}\right)(1+b)}{p_{0}\left[1+b\left(n_{0} / p_{0}\right)\right]}\left[1-e^{-t / \tau}\right]
$$

darstellen läßt. Es bedeuten: $\tau$ gesuchte Lebensdauer, $R$ Widerstand des Kristalls, $n\left(t_{1}\right)$ Zahl der injizierten Elektronen, die z. Zt. $t_{1}$ beim Abschalten des ersten Impulses vorhanden sind, $p_{0}$ und $n_{0}$ Dichte der im Gleichgewicht vorhandenen Defektelektronen und Elektronen und $b=\mu_{\mathrm{n}} / \mu_{\mathrm{p}}=$ Beweglichkeitsverhältnis.

Die Methode ist mit derjenigen von Navon, Bray und FAN verwandt ${ }^{7}$. Der Vorteil des Doppelimpulsverfahrens liegt darin, daß nach Abschalten des ersten Impulses die Änderung des Widerstands auf Grund der Rekombination von Minoritätsladungsträgern nicht kontinuierlich gemessen wird, und somit keine Korrekturen für Ladungsträger, die während der Widerstandsmessung den Kristall verlassen haben, anzubringen sind. Denn das Maß für den zur Zeit $t$ vorhandenen Widerstand ist die Höhe des zweiten Impulses unmittelbar nach dessen Einsetzen, der weitere Verlauf (bedingt durch die neu einsetzende Injektion) ist für die Lebensdauermessung uninteressant. Es ist möglich, die Lebensdauer auf dem Oszillographenschirm direkt abzulesen, wenn man die Verzögerungszeit so wählt, daß die Höhe des zweiten Impulses das $(1-1 / e)$-fache des ersten beträgt. Das gilt jedoch nur, wenn die Meßkristalle gut geätzt und so groß sind, daß ein anfänglicher steilerer Anstieg des Widerstands auf Grund von Oberflächenrekombination nicht ins Gewicht fällt. In

7 D. NAvon, R. Bray u. H. Y. FAN, Proc. Inst. Radio Engrs 40, 1342 [1952]. 
allen übrigen Fällen wird die Lebensdauer aus einer logarithmischen Auftragung der Impulshöhendifferenz über der Zeit ermittelt. Die Genauigkeit der Doppelimpulsmethode ist etwa gleich groß wie die der Methode von Many. Bei Vergleichsmessungen konnten innerhalb der Meßunsicherheit von etwa $\pm 5 \%$ keine Abweichungen in den Ergebnissen festgestellt werden. Abb. 4 zeigt ein typisches Oszillogramm.

\section{Kristallmaterial}

Zur Messung der Löcherdriftbeweglichkeit mit der Methode von Lawrence und Gibson wurden 3 homogene, senkrecht zur Ziehrichtung geschnittene Einkristalle aus $\mathrm{n}$-Germanium der Abmessungen $0,4 \times 0,6 \times 12 \mathrm{~mm}^{3}$ benutzt. Das Ausgangsmaterial hatte $300 \mu \mathrm{s}$ Volumenlebensdauer ${ }^{8}$, nach Ätzung mit $\mathrm{CP} 4$ und längerer Lagerung an Luft ergab sich eine Oberflächenrekombinationsgeschwindigkeit von ca. $500 \mathrm{~cm} / \mathrm{s}$. Zwei Kristalle hatten einen spezifischen Widerstand von $11 \Omega \mathrm{cm}$ (N1 und N2), der dritte $2 \Omega \mathrm{cm}$ (N3). Ein hochohmiger Kristall besaß als Emitter und Kollektor anlegierte Indiump n-Übergänge, die anderen beiden Exemplare waren mit verschweißten $50 \mu$ Golddrähten versehen. Der Abstand von Emitter und Kollektor betrug einige Millimeter. Die Endkontakte waren sperrfreie $\mathrm{n}^{+} \mathrm{n}$-Übergänge. Bei allen verwendeten Kristallen waren zur Vermeidung mechanischer Spannungen die Zuführungsdrähte federnd angebracht. Der n-Kristall (N4), der zur Messung der Driftbeweglichkeit mit der Brückenmethode benutzt wurde, war mit Antimon dotiert und hatte $80 \mu$ s Volumenlebensdauer und nach Ätzung mit Natriumhypochloritlösung eine Oberflächenrekombinationsgeschwindigkeit von $145 \mathrm{~cm} / \mathrm{s}$. Die Abmessungen betrugen $11,2 \times 1,5 \times 1,5 \mathrm{~mm}^{3}$, der spezifische Widerstand $11 \Omega \mathrm{cm}$. Die Endkontakte waren $\mathrm{Sn}-\mathrm{As}$-Lötungen. $\mathrm{Zu}$ Widerstandsmessungen wurden ferner Antimon-dotierte Kristalle mit $5 \Omega \mathrm{cm}$ und $2,9 \Omega \mathrm{cm}$ (N5 und N6) spezifischem Widerstand verwendet. Das p-Germanium, aus dem die Kristalle zur Messung der Elektronendriftbeweglichkeit geschnitten wurden, hatte eine Volumenlebensdauer von $120 \mu$ s. Eine Probe mit den Abmessungen $10,2 \times 1,2 \times 1,0 \mathrm{~mm}^{3}(\mathrm{Pl})$, die am eingehendsten untersucht wurde, besaß nach Ätzen mit CP 4 eine Oberflächenrekombinationsgeschwindigkeit von $320 \mathrm{~cm} / \mathrm{s}$. Die Endkontakte waren mit Indium angelötet.

\section{Gang der Messungen und Meßergebnisse}

Bei allen Widerstands- und Beweglichkeitsmessungen wurden die Dimensionsänderungen des Germaniums durch Druckeinwirkung korrigiert. Bei der Bestimmung der Löcherbeweglichkeit in den n-Kri-

8 E. Arends, persönliche Mitteilung.

- M. B. Prince, Phys. Rev. 91, 271 [1953]. stallen mit dem spezifischen Widerstand $11 \Omega \mathrm{cm}$ wurde der Einfluß der im Gleichgewicht vorhandenen Löcher auf die Impuls-Laufzeit ${ }^{9}$ gemäß der Beziehung

$$
\mu_{\mathrm{D}} / \mu_{\mathrm{g}}=1+\frac{1}{2}(1+1 / b)\left[\left(1+4 n_{\mathrm{i}}^{2} / N_{\mathrm{D}}^{2}\right)^{1 / 2}-1\right]
$$

$\mu_{\mathrm{D}}=$ Löcherdriftbeweglichkeit, $\mu_{\mathrm{g}}=$ gemessene Beweg. lichkeit des Löcherimpulses, $b=\mu_{\mathrm{n}} / \mu_{\mathrm{p}}, \quad n_{\mathrm{i}}=$ Eigenleitungskonzentration, $N_{\mathrm{D}}=$ Donatorenkonzentration.

berücksichtigt. $n_{\mathrm{i}}^{2} / N_{D}^{2}$ wurde in Abhängigkeit vom Druck am Kristall N4 gemessen, wovon im folgenden noch berichtet wird.

Von den Ergebnissen der Messung mit der Methode von Lawrence und Gibson wurde bereits kurz berichtet ${ }^{10}$. In Abb. 5 ist für den Kristall $\mathrm{Nl}$ mit

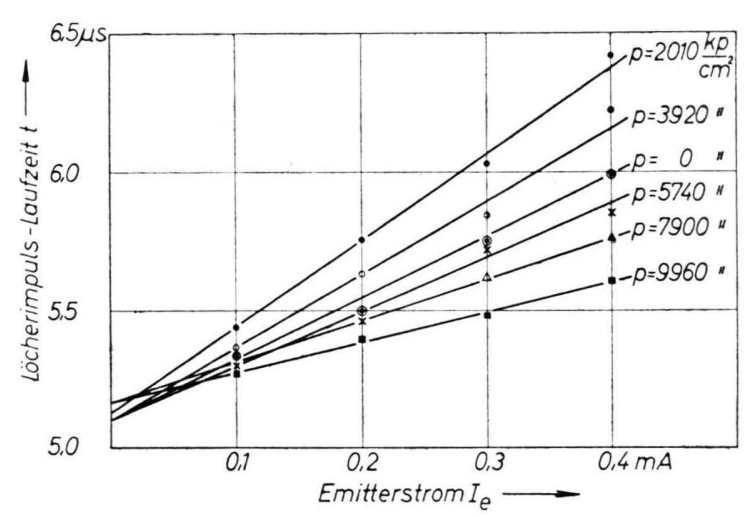

Abb. 5. Laufzeit des Löcherimpulses in Abhängigkeit vom Emitterstrom mit dem Druck als Parameter.

Indiumemitter und -kollektor die Abhängigkeit der Laufzeit des Löcherimpulses von der Emitterstromstärke mit dem Druck als Parameter aufgetragen, bei konstanter Feldstärke und einer Temperatur von $22{ }^{\circ} \mathrm{C}$. Bei der Berechnung der Beweglichkeit sind noch die relativ kleinen Korrekturen für die Längenänderungen unter Druck und der Impulsanstiegszeit anzubringen. Die Berücksichtigung von (2) ergibt eine Korrektur von $1,7 \%$ bei $p=0$, die mit zunehmendem Druck rasch abnimmt. Das Ergebnis ist für die drei untersuchten Kristalle charakteristisch. Alle Extrapolationsgeraden münden etwa an einer Stelle. Innerhalb der Meßunsicherheit von ca. $\pm 2 \%$ ist die Löcherbeweglichkeit druckunabhängig. Die Meßsicherheit reichte nicht aus zu entscheiden, ob sich innerhalb der angegebenen Grenzen die Beweglichkeit mit dem Druck ändert. Alle drei untersuchten

10 G. Landwehr, Z. Naturforschg. 11 a, 257 [1956]. 
Kristalle zeigten hinsichtlich der Druckabhängigkeit der Beweglichkeit ein im wesentlichen gleiches Verhalten, lediglich bei dem Exemplar mit legierten Kontakten trat eine leichte Hysterese auf. Es zeigte sich, daß die Steigung der Extrapolationsgeraden druckabhängig war. Aus diesem Grunde konnte man sich nicht darauf beschränken, die Beweglichkeitsänderung bei nur einer Emitterstromstärke zu messen. Die Steigung der Extrapolationsgeraden ist für Auf- und Abwärtsgang in Abb. 6 dargestellt. Bemer-

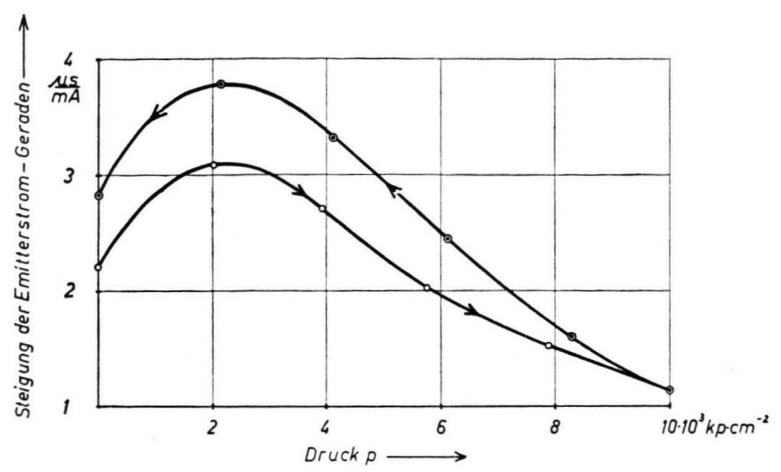

Abb. 6. Steigung der Emitterstromgeraden in Abhängigkeit vom Druck für Auf- und Abwärtsgang.

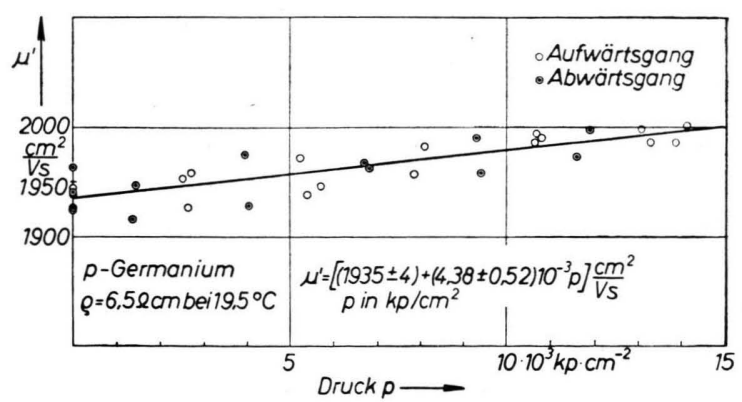

Abb. 7. Löcherdriftbeweglichkeit in Germanium $\mu^{\prime}$ in Abhängigkeit vom Druck, ohne Kompressibilitätskorrektur.

kenswert ist das Maximum bei ca. $2000 \mathrm{kp} / \mathrm{cm}^{2}$ und die ausgeprägte Hysterese, die sich auch durch mehrmalige Druckanwendung nicht beseitigen ließ. Auch bei den mit Goldkontakten versehenen Exemplaren wurden in der Steigung der Extrapolationsgeraden Maxima und Hysteresiserscheinungen beobachtet, die allerdings nicht so ausgeprägt waren. Die Effekte können durch Annahme einer Änderung der Oberflächenrekombinationsgeschwindigkeit gedeutet werden. Die erzielten Meßergebnisse sollen hier aber nicht weiter diskutiert werden, da die Versuche mit der Brückenmethode größeres Gewicht haben.

Um den statischen Fehler herabzudrücken, wur- den mit dem Brückenverfahren an dem $11 \Omega \mathrm{cm}$-Kristall N4 insgesamt drei Meßreihen der Druckabhängigkeit der Löcherbeweglichkeit bei Drucken bis zu $14000 \mathrm{kp} / \mathrm{cm}^{2}$ ausgeführt, bei einer Temperatur von $(19,5 \pm 0,1){ }^{\circ} \mathrm{C}$. Im Aufwärtsgang wurde in Stufen von rund $2500 \mathrm{kp} / \mathrm{cm}^{2}$ gemessen, im Abwärtsgang der Druck zunächst um $1250 \mathrm{kp} / \mathrm{cm}^{2}$, dann um jeweils $2500 \mathrm{kp} / \mathrm{cm}^{2}$ erniedrigt. Der maximal hergestellte Druck war durch das Festwerden der druckübertragenden Flüssigkeit bedingt. Bei jedem Druck wurden 5 Spannungsmessungen gemacht und der Mittelwert benutzt. Das Ergebnis der Messungen ist in Abb. 7 dargestellt. Die Korrekturen nach Gl. (2) sind berücksichtigt, die Dimensionsänderungen durch Druckeinfluß noch nicht, die Resultate sind also direkt mit Leitfähigkeitsmessungen vergleichbar. Eine Ausgleichsrechnung ergab

$\mu^{\prime}(p)=\left[(1935 \pm 4)+(4,38 \pm 0,52) 10^{-3} p\right] \mathrm{cm}^{2} /$ Vs.

Wenn man die Meßwerte mit dem Faktor $l(p)^{2} / l(0)^{2}$ multipliziert, wobei $l$ die Länge des Kristalls bedeutet, erhält man die Änderung der Löcherdriftbeweglichkeit $\mu$. Für die prozentuale Änderung der Löcherdriftbeweglichkeit wurde so gefunden:

$$
\begin{gathered}
\mu(p) / \mu(0)=(1 \pm 0,0021)+(1,39 \pm 0,27) 10^{-6} p, \\
(p \text { in kp/cm }) .
\end{gathered}
$$

Die angegebenen Fehler sind die Standardabweichungen. Sie gelten nur für die Relativmessung der Änderung der Beweglichkeit durch hydrostatischen Druck. Bei diesen Messungen wurde stets nur ein ziemlich enger Bereich benutzt, so daß Fehler in der Kalibrierung der Zeitskala und des Voltmeters nur unwesentlich ins Gewicht fallen. Die absolute Meßunsicherheit, welche den möglichen Fehler bei der Bestimmung der Länge des Kristalls einschließt, dürfte kleiner als $2 \%$ sein. Nicht leicht zu beurteilen ist die Unsicherheit, die durch Anbringung der Korrekturen nach Gl. (2) entsteht. Die Messungen von Prince ${ }^{9}$ rechtfertigen jedoch einiges Zutrauen in die Gültigkeit der Korrektur. Die Ungenauigkeit in der Druckmessung, die max. 1\% erreichen kann, ist ohne Einfluß auf das Meßergebnis. Fehler, die durch Abweichungen vom Temperaturgleichgewicht entstehen können, sind kleiner als 0,8\% $\%$. Der Absolutwert der Löcherdriftbeweglichkeit von $(1935 \pm 40) \mathrm{cm}^{2} / \mathrm{Vs}$ bei $T=292,7^{\circ} \mathrm{K}$ entsprechend $(1827 \pm 37) \mathrm{cm}^{2} / \mathrm{Vs}$ bei $T=300^{\circ} \mathrm{K}$ stimmt mit dem Wert von Prince überein, der für $\mu_{\mathrm{D}}$ bei dieser Temperatur (1800 $\pm 54) \mathrm{cm}^{2} / \mathrm{Vs}$ findet. 
In Abb. 8 ist die Änderung der Leitfähigkeitsbeweglichkeit mit dem Druck aufgetragen, abgeleitet aus einer Widerstandsmessung an dem p-GermaniumKristall PI mit einem spezifischen Widerstand von $6,5 \Omega \mathrm{cm}$. Effekte durch noch vorhandene Eigenleitungselektronen treten nicht auf, was aus dem linea-

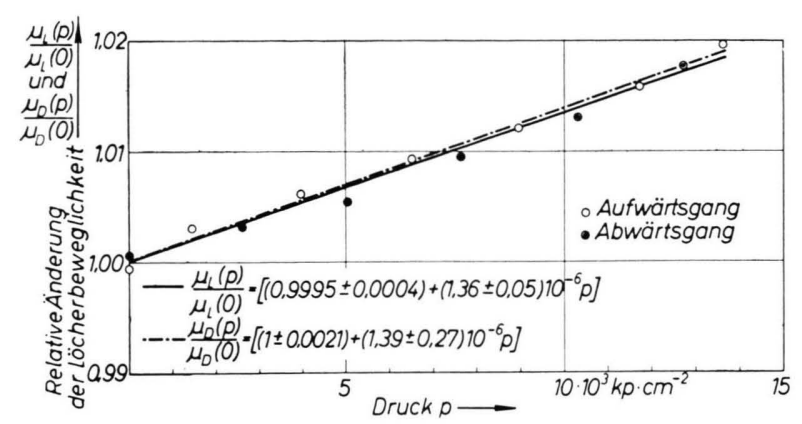

Abb. 8. Relative Änderung der Löcherleitfähigkeitsbeweglichkeit (ausgezogene Gerade) und der Löcherdriftbeweglichkeit (gestrichelte Gerade) in Germanium in Abhängigkeit vom Druck.

ren Verlauf der Kurve zu ersehen ist. Die Streuungen der Meßwerte sind vorwiegend auf Temperaturschwankungen zurückzuführen. Die Übereinstimmung mit der Messung der Löcherdriftbeweglichkeit in Abhängigkeit vom Druck (gestrichelte Gerade) ist recht gut. Die durchgehend ausgezogene Gerade wurde ebenfalls durch Ausgleichsrechnung ermittelt, für die prozentuale Änderung der Leitfähigkeitsbeweglichkeit in Abhängigkeit vom Druck ergab sich:

$$
\begin{aligned}
\mu_{\mathrm{L}}(p) / \mu_{\mathrm{L}}(0)= & (0,99995 \pm 0,0004) \\
+ & (1,36 \pm 0,05) \cdot 10^{-6} p, \\
& \left(p \text { in } \mathrm{kp} / \mathrm{cm}^{2}\right) .
\end{aligned}
$$

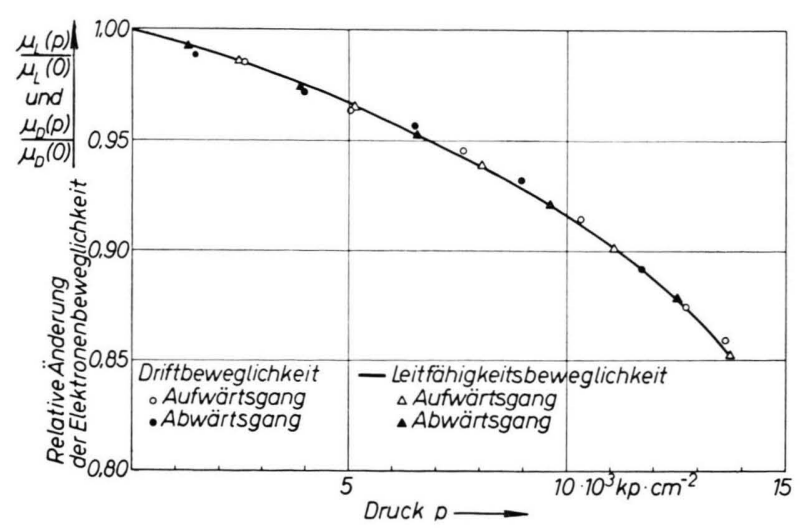

Abb. 9. Relative Änderung der Driftbeweglichkeit und der Leitfähigkeitsbeweglıchkeit von Elektronen in Girmaıım in Abhängigkeit vom Druck.
Der angegebene Fehler ist die Standardabweichung.

Abb. 9 zeigt das Ergebnis einer Messung der Druckabhängigkeit der Elektronendriftbeweglichkeit im Kristall P1. Bei jedem eingestellten Druck wurde bei vier verschiedenen Injektionsverhältnissen gemessen und durch eine Ausgleichsgerade auf die Injektion 0 extrapoliert. Bei konstanter Injektion wurden jeweils vier Feldstärkemessungen vorgenommen. Dadurch ist es zu erklären, daß die Streuung der Meßwerte so gering ist. Impuls-Driftkorrekturen wurden nicht angebracht, da sie gemäß einer Gl. (2) analogen Formel so klein waren, daß sich eine Berücksichtigung erübrigte, was durch die Widerstandsmessungen am Kristall P1 bestätigt wurde. Die Abnahme der Driftbeweglichkeit von Elektronen im p-Germanium mit zunehmendem Druck, die durch eine Messung an einem weiteren p-Kristall (P2) mit dem spezifischen Widerstand $5,3 \Omega \mathrm{cm}$ bestätigt wurde, ist in guter Übereinstimmung mit der Änderung der Leitfähigkeitsbeweglichkeit bei Druckanwendung, die aus der Widerstandsänderung des $2,9 \Omega \mathrm{cm}$-Kristalls N6 abgeleitet wurde und die aus derselben Abbildung zu entnehmen ist. Für die Meßunsicherheit gilt analog das bei n-Germanium Gesagte, die relative Meßgenauigkeit dürfte bei der Bestimmung der Elektronendriftbeweglichkeit in Abhängigkeit vom Druck etwas höher sein. Der Absolutwert der Beweglichkeit, der an den Kristallen P1 und P2 gemessen wurde, liegt mit $3450 \mathrm{~cm}^{2} / \mathrm{Vs}$ bei $295,2{ }^{\circ} \mathrm{K}$ zu niedrig. Der Grund hierfür konnte nicht ermittelt werden. Eine Kompensation von $\mathrm{Ak}$ zeptoren, die Anlaß zu einer erhöhten Streuung an ionisierten Störstellen geben könnte, ist ausgeschlossen, da das Kristall-Ausgangsmaterial fast eigenleitend war und mit Gallium dotiert wurde ${ }^{11}$.

Als typisch für die ausgeführten Lebensdauermessungen von Minoritätsladungsträgern sind die Ergebnisse für den Kristall Pl in Abb. 10 dargestellt, und zwar für drei Druckanwendungen. Bei der ersten Druckbelastung (Kurve I) stieg die effektive Elektronenlebensdauer zunächst an, um bei höheren Drucken abzufallen. Im Abwärtsgang lag die Lebensdauer ab ca. $6000 \mathrm{kp} / \mathrm{cm}^{2}$ über den im Aufwärtsgang gemessenen Werten und sank im Laufe von 4. Tagen auf den Anfangswert der mit II bezeichneten Kurve. Auch beim zweiten Druckzyklus zeigten sich ein Maximum und ausgeprägte Hysterese. Nach einigen Tagen war die Lebensdauer abermals gesun-

11 E. Arends, persönliche Mitteilung. 


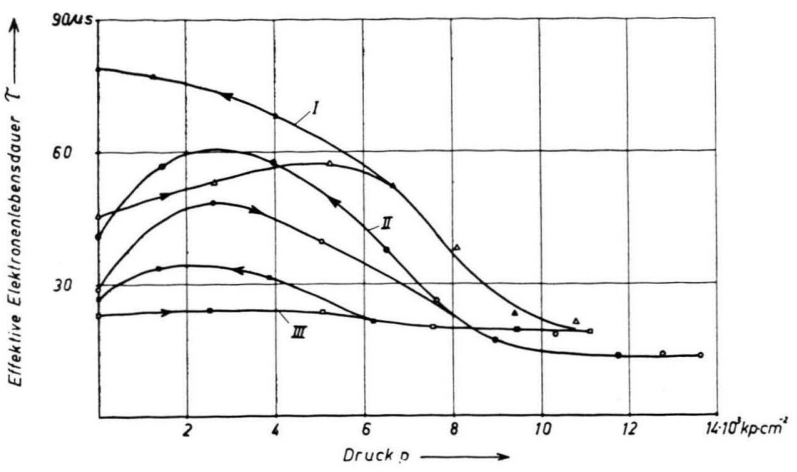

Abb. 10. Effektive Lebensdauer von Elektronen in p-Germanium in Abhängigkeit vom Druck bei dreimaliger Druckanwendung, jeweils für Auf- und Abwärtsgang.

ken und änderte sich bei der nächsten Druckanwendung gemäß Kurve III. Danach wurde der Kristall ausgebaut und mit frischer Druckflüssigkeit gespült, wodurch die effektive Lebensdauer wieder auf $60 \mu \mathrm{s}$ gebracht wurde. Eintauchen in Dekalin ergab eine Lebensdauer von $40 \mu \mathrm{s}$; damit dürfte erwiesen sein, daß die beobachteten Änderungen zumindest z. Tl. auf Oberflächeneffekten beruhen. Mechanische Veränderungen oder Störungen an der Oberfläche der Versuchsprobe konnten nicht festgestellt werden. Eine Deutung der Erscheinungen kann z. Zt. noch nicht gegeben werden. Die Driftbeweglichkeitsmessungen wurden von den Lebensdaueränderungen nicht beeinflußt.

\section{Bestimmung der Eigenleitungskonzentration und des Druckkoeffizienten der verbotenen Zone}

Die Eigenleitungskonzentration $n_{\mathrm{i}}$ in Germanium, deren Kenntnis erforderlich ist, um aus der gemessenen Impulsdriftbeweglichkeit die Löcherdriftbeweglichkeit zu ermitteln ${ }^{9}$, wurde aus Druckmessungen bestimmt. Dabei ergab sich auch der Druckkoeffizient der verbotenen Zone.

Zur Messung von $n_{\mathrm{i}}$ wurden drei Kristalle aus nGermanium (N4, N5, N6) mit den spezifischen Widerständen $11 \Omega \mathrm{cm}, 5,0 \Omega \mathrm{cm}$ und $2,9 \Omega \mathrm{cm}$ in die Apparatur eingebaut und gleichzeitig gedrückt. Die prozentualen Widerstandsänderungen sind in Abb. 11 dargestellt. Es ist deutlich zu sehen, daß der Kristall N4 auf Grund seines Eigenleitungsanteils, der mit wachsendem Druck exponentiell abnimmt, seinen Widerstand stärker ändert als die höherdotierten Proben N5 und N6. Bildet man die Differenzen der Widerstandsänderungen von zwei

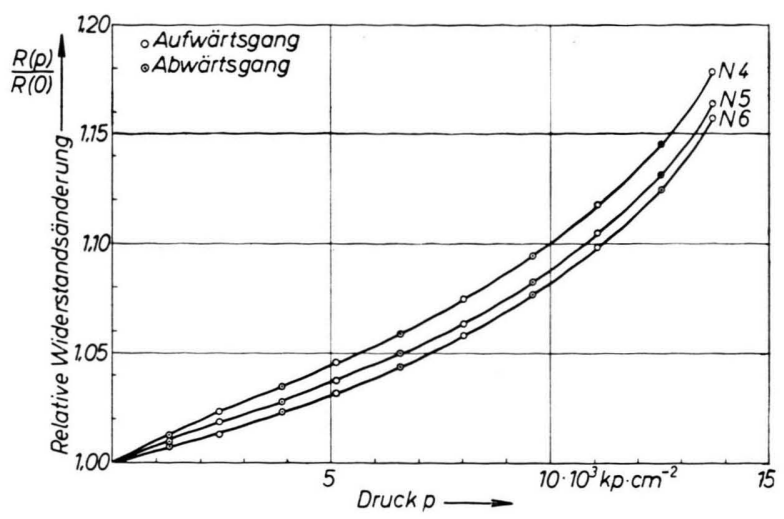

Abb. 11. Relative Widerstandsänderung von $3 \mathrm{n}$-GermaniumKristallen mit den spezifischen Widerständen $2,9 \Omega \mathrm{cm}, 5 \Omega \mathrm{cm}$ und $11 \Omega \mathrm{cm}$ in Abhängigkeit vom Druck.

Versuchsexemplaren, eliminiert man also die Widerstandsänderung auf Grund von Beweglichkeitsänderungen durch Druckeinfluß, ist es möglich, daraus die Eigenleitungskonzentration zu bestimmen. Das Verfahren hat zur Voraussetzung, daß die Ladungsträgerbeweglichkeit von beiden Proben durch den gleichen Streumechanismus bedingt ist, was bei den Kristallen N4 und N5 der Fall ist. Bei diesen Exemplaren wird die Beweglichkeit durch die Streuung der Leitungselektronen an den akustischen Gitterschwingungen bedingt, Streuung an ionisierten Störstellen kann vernachlässigt werden. Da beim Kristall N6 der zuletzt erwähnte Mechanismus nicht ganz außer Betracht bleiben kann, wurde diese Messung der Widerstandsänderung nicht zur Bestimmung von $n_{\mathrm{i}}$ herangezogen.

Unter der Voraussetzung, daß die Akzeptorenkonzentration unberücksichtigt bleiben kann, was gerechtfertigt ist, da das Ausgangsmaterial der verwendeten Kristalle eigenleitend war, gilt bei Zimmertemperatur

$$
\begin{aligned}
n-p & =N_{\mathrm{D}} \text { und mit } n p=n_{\mathrm{i}}{ }^{2}: \\
n & =\frac{1}{2} N_{\mathrm{D}}\left[\left(1+4 n_{\mathrm{i}}{ }^{2} / N_{\mathrm{D}}\right)^{1 / 2}+1\right], \\
p & =\frac{1}{2} N_{\mathrm{D}}\left[\left(1+4 n_{\mathrm{i}}{ }^{2} / N_{\mathrm{D}}\right)^{1 / 2}-1\right] ;
\end{aligned}
$$

$N_{\mathrm{D}}=$ Konzentration der Donatoren, $n=$ Elektronenkonzentration, $p=$ Löcherkonzentration, $n_{\mathrm{i}}=$ Eigenleitungs. konzentration.

Ist $n_{\mathrm{i}}{ }^{2} \ll N_{\mathrm{D}}{ }^{2}$, gilt in erster Näherung

$$
n=N_{\mathrm{D}}\left[1+\left(n_{\mathrm{i}}^{2} / N_{\mathrm{D}}^{2}\right)\right] \text { und } p=n_{\mathrm{i}}^{2} / N_{\mathrm{D}} .
$$

Die spezifische Leitfähigkeit beträgt dann:

$$
\sigma(0)=N_{\mathrm{D}} e \mu_{\mathrm{n}}(0)\left[1+\frac{n_{\mathrm{i}}^{2}(0)}{N_{\mathrm{D}^{2}}}\left(1+\frac{1}{b(0)}\right) / N_{\mathrm{D}}^{2}\right]
$$

$b=\mu_{\mathrm{n}} / \mu_{\mathrm{p}}=$ Beweglichkeitsverhältnis. 
Das Argument (0) weist auf den Überdruck 0 hin. Wegen der Druckabhängigkeit von $\mu, n_{\mathrm{i}}$ und $b$ und der Druckunabhängigkeit von $N_{\mathrm{D}}$ gilt bei dem Druck $p$ :

$$
\sigma(p)=N_{\mathrm{D}} e \mu_{\mathrm{n}}(p)\left[1+\frac{n_{\mathrm{i}}^{2}(p)}{N_{\mathrm{D}}{ }^{2}}\left(1+\frac{1}{b(p)}\right)\right] .
$$

Mit $\varrho=\sigma^{-1}$ erhält man für die prozentuale Änderung des spezifischen Widerstandes für einen Kristall (1) mit der Donatorenkonzentration $N_{\mathrm{D}}$ :

$$
\left(\frac{\varrho(p)-\varrho(0)}{\varrho(0)}\right)_{1}=\frac{\mu_{\mathrm{n}}(0)\left[1+\frac{n_{\mathrm{i}}^{2}(0)}{N_{\mathrm{D}}^{2}}\left(1+\frac{1}{b(0)}\right)\right]}{\mu_{\mathrm{n}}(p)\left[1+\frac{n_{\mathrm{i}}^{2}(p)}{N_{\mathrm{D}}{ }^{2}}\left(1+\frac{1}{b(p)}\right)\right]}-1 .
$$

Für einen zweiten Kristall (2), dessen Donatorenkonzentration $k N_{\mathrm{D}}$ beträgt, gilt eine analoge Formel. Als Differenz der prozentualen Widerstandsänderungen zweier Kristalle mit den Donatorenkonzentrationen $N_{\mathrm{D}}$ und $k N_{\mathrm{D}}$ ergibt sich unter Vernachlässigung von Gliedern höherer Ordnung

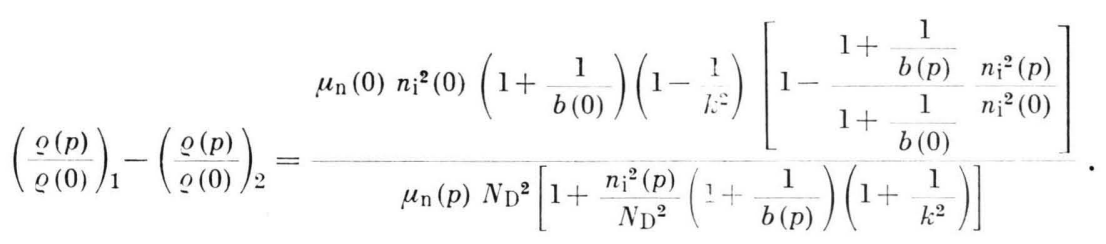

\section{Setzt man}

$$
n_{i}{ }^{2}(p)=4 A^{2} \exp \left\{-\left[E_{\mathrm{g}}+\left(\partial E_{\mathrm{g}} / \partial p\right)_{T} \cdot p\right] / k T\right\},
$$

wobei $A$ von der Temperatur und der effektiven
Masse abhängt, und läßt Änderungen der effektiven Masse durch Druckeinfluß außer Betracht, erhält man schließlich

$$
\left(\frac{\varrho(p)}{\varrho(0)}\right)_{1}-\left(\frac{\varrho(p)}{\varrho(p)}\right)_{2}=\frac{\mu_{\mathrm{n}}(0) n_{\mathrm{i}}{ }^{2}(0)\left(1+\frac{1}{b(0)}\right)\left(1-\frac{1}{k^{2}}\right)\left[1-\frac{1+\frac{1}{b(p)}}{1+\frac{1}{b(0)}} \exp \left\{-\left[\left(\partial E_{\mathrm{g}} / \partial p\right) T \cdot p\right] / k T\right\}\right.}{\mu_{\mathrm{n}}(p) N_{\mathrm{D}^{2}}\left[1+\frac{n_{\mathrm{i}}{ }^{2}(p)}{N_{\mathrm{D}}{ }^{2}}\left(1+\frac{1}{b(p)}\right)\left(1+\frac{1}{k^{2}}\right)\right]} .
$$

$N_{\text {D }}$ und $k$ sind aus Leitfähigkeitsmessungen und den geometrischen Abmessungen der Kristalle bekannt, $\mu_{\mathrm{n}}(p)$ und $b(p)$ aus Messungen der Druckabhängigkeit der Driftbeweglichkeit. Somit können $n_{\mathrm{i}}{ }^{2}(0)$ und $\left(\partial E_{\mathrm{g}} / \partial p\right)_{T}$ bestimmt werden. Auf die Berücksichtigung des Faktors $[1+1 / b(p)] /[1+1 / b(0)]$ konnte verzichtet werden, da er bei niedrigen Drucken

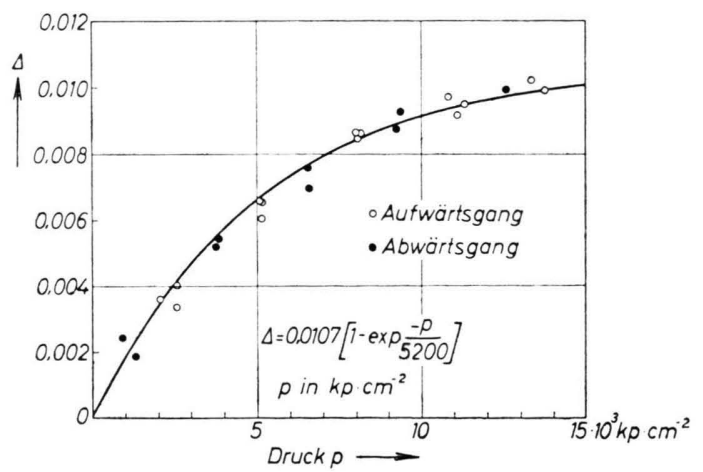

Abb. 12. Differenz der relativen Widerstandsänderung eines $5 \Omega \mathrm{cm}$ - und eines $11 \Omega \mathrm{cm}$-n-Germanium-Kristalls in Abhängigkeit vom Druck, korrigiert zur Ermittlung von $n_{\mathrm{i}}^{2}$ und des Druckkoeffizienten der verbotenen Zone. nur wenig von 1 verschieden ist und bei höheren der Einfluß des Exponentialfaktors überwiegt. Es war auch nicht erforderlich, die gemessenen Widerstände in spezifische umzurechnen und die Kompressibilitätskorrektur anzubringen; wegen der Differenzbildung lagen diese Einflüsse weit unterhalb der Fehlergrenze. Auch das zweite Glied des Nenners von (3) blieb außer Betracht, da $n_{\mathrm{i}}^{2} \ll N_{\mathrm{D}}^{2}$ war und überdies mit zunehmendem Druck exponentiell abnimmt. In Abb. 12 ist die durch die Faktoren

$$
\left(1-1 / K^{2}\right)(1+1 / b(0)) \mu_{\mathrm{n}}(0) / \mu_{\mathrm{n}}(p)
$$

dividierte Differenz der prozentualen Widerstandsänderungen aufgetragen. Dargestellt ist das Resultat von drei Meßreihen. Bei einer waren die Streuungen höher als bei den übrigen, die ausgezogene Kurve wurde entsprechend dem Gewicht der einzelnen Messungen eingezeichnet. Die Anforderungen an die Präzision der Widerstandsmessung sind verhältnismäßig hoch, da Meßfehler sich wegen der Differenzbildung empfindlich bemerkbar machen. Die Temperaturkonstanz wurde mittels des eingebauten Platin- 
Widerstandsthermometers überwacht. Temperaturschwankungen blieben unter 0,05 Grad. Für die Widerstände beim Druck 0 wurde das arithmetische Mittel aus jeweils sechs Messungen verwendet. Die relativen mittleren Abweichungen des Null-Widerstandes betrugen $5 \cdot 10^{-5}$ bei Kristall $\mathrm{N} 4$ und $6,7 \cdot 10^{-4}$ bei $\mathrm{N} 5$. Die Meßwerte lassen sich am besten durch die Funktion

$$
\Delta=0,0107(1-\exp \{-p / 5200\})
$$

approximieren. Daraus entnimmt man für $n_{\mathrm{i}}{ }^{2} / N_{\mathrm{D}}{ }^{2}$ den Wert 0,0107. Bei Annahme einer Elektronenbeweglichkeit von $3800 \mathrm{~cm}^{2} / \mathrm{Vs}$ ergibt sich bei $T=292,7{ }^{\circ} \mathrm{K}$ für $n_{\mathrm{i}}{ }^{2}$ der Wert $2,41 \cdot 10^{26} / \mathrm{cm}^{3}$. Dadurch, da $\beta N_{\text {D }}$ aus dem spezifischen Widerstand des Kristalls und dem Literaturwert für die Beweglichkeit bestimmt wurde, ist der mögliche Fehler ziemlich groß. Zwar wurden die Meßkristalle sorgfältig planparallel geschliffen, so daß nach dem Ätzen die Abweichungen von der Planparallelität kleiner als $0,01 \mathrm{~mm}$ waren, und die Unsicherheit des spezifischen Widerstandes etwa $2 \%$ beträgt. Mit ca. $\pm 3 \%$ Unsicherheit der Beweglichkeit und rund $3 \%$ des Wertes für $n_{\mathrm{i}}^{2} / N_{\mathrm{D}}{ }^{2}$ ergibt sich ein möglicher Gesamtfehler von etwa $\pm 13 \%$. Innerhalb dieser Grenzen besteht Übereinstimmung mit $n_{\mathrm{i}}{ }^{2}=2,59 \cdot 10^{26} / \mathrm{cm}^{3}$ nach Morin und Maita ${ }^{12}$ bei $T=292,7^{\circ} \mathrm{K}$. Der Wert $2,89 \cdot 10^{26} / \mathrm{cm}^{3}$ für $n_{\mathrm{i}}{ }^{2}$ nach Conweld ${ }^{13}$ liegt dagegen außerhalb der Fehlergrenzen.

Als Druckkoeffizienten der verbotenen Zone bei $T=292,7^{\circ} \mathrm{K}$ ermittelt man aus Abb. 12 den Wert $\partial E_{\mathrm{g}} / \partial p=4,9 \cdot 10^{-6} \mathrm{eV} \mathrm{cm} / \mathrm{kp}$. Die geschätzte Meß-

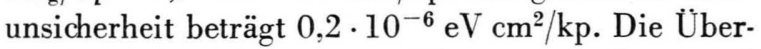
einstimmung mit neueren Werten von PAUL und Brooks $^{14}$, sowie NAthan ${ }^{15}$, die $\partial E_{\mathrm{g}} / \partial p=5,0 \cdot 10^{-6}$ $\mathrm{eV} \mathrm{cm} / \mathrm{kp}$ bei ca. $350^{\circ} \mathrm{K}$ finden, ist befriedigend.

\section{Diskussion und Deutung der Meßergebnisse}

Die Messungen zeigen, daß mit wachsendem Druck die Löcherbeweglichkeit in Germanium schwach zunimmt und daß Übereinstimmung zwischen der Änderung der Driftbeweglichkeit von injizierten Löchern in n-Germanium und der Änderung der Leitfähigkeitsbeweglichkeit von Löchern in p-Germanium besteht. Daraus kann man schließen, daß die Zahl

12 F. J. Morin u. J. P. Maita, Phys. Rev. 94, 1525 [1954].

13 E. M. Conwell, Proc. Inst. Radio Engrs 40, 1327 [1952].

14 W. Paul u. H. Brooks, Phys. Rev. 94, 1128 [1954]. der Ladungsträger, die zur Leitfähigkeit beitragen, durch Anwendung hoher Drucke nicht beeinflußt wird. Die Ursache der Widerstandsabnahme von p-Germanium unter Druck ist in einer Zunahme der Ladungsträgerbeweglichkeit zu suchen und nicht in einer Änderung des Ionisationszustandes der Akzeptoren. Für diese Deutung spricht auch die Reproduzierbarkeit der Messungen. Die Möglichkeit einer Verfälschung der Driftbeweglichkeitsmessungen durch Haftstellen ist ziemlich ausgeschlossen. Haftstellen mit einer Löcherverweilzeit, die mit der Laufzeit im Kristall vergleichbar ist, hätten sich bei der Methode von Lawrence und Gibson durch eine Impulsverformung bemerkbar gemacht. Derartige Effekte wurden jedoch nicht beobachtet. Haftstellen mit kurzer Verweilzeit müßten für Löcher in n- und p-Germanium dieselbe Druckabhängigkeit des Widerstandes hervorrufen, was sehr unwahrscheinlich ist. Überdies ist bekannt, daß im allgemeinen Haftstellen bei Germanium (im Gegensatz zu Silicium) bei Zimmertemperatur keine Rolle spielen. Zieht man Haftstellen, die durch Gitterstörungen in Form von Versetzungen bedingt sind, in Betracht, ist zu bedenken, daß die elastischen Spannungen der unmittelbaren Umgebung einer Versetzung wesentlich höher sind als die angewendeten hydrostatischen Drucke, so daß eine Erzeugung von Haftstellen durch Druckanwendung nicht wahrscheinlich ist. Die Übereinstimmung der indirekten Meßmethode mit der fundamentalen, die auf einer Weg-, Zeit- und Spannungsmessung beruht, ist recht befriedigend.

Die untersuchten Germaniumkristalle waren so dotiert, daß die Ladungsträgerbeweglichkeit durch Streuung an den Gitterschwingungen bedingt war; Streuung an ionisierten Störstellen und Versetzungen konnte außer Betracht bleiben. Zur Deutung der Druckabhängigkeit der Löcherdriftbeweglichkeit kann man die Deformationspotentialtheorie von BARDEEN und SHockley ${ }^{16}$ unter gewissen Vorbehalten heranziehen.

Für die Gitterbeweglichkeit gilt nach ${ }^{16}$

$$
\mu_{\mathrm{p}}=\frac{\sqrt{8 \pi}}{3} \frac{\hbar^{4}}{E_{1 \mathrm{p}}^{2}} \frac{c_{\mathrm{ii}} T^{-3 / 2}}{m_{\mathrm{p}}{ }^{* 5 / 2} k^{3 / 2}}
$$

$\hbar=1 / 2 \pi$ Plancksches Wirkungsquantum,
$k=$ Boltzmans-Konstante, $T=$ absolute Temperatur

15 M. I. Nathan, Technical Report No. HPl (1958), Gordon McKay Laboratory, Harvard University.

16 J. Bardeen u. W. Shockley, Phys. Rev. 80, 72 [1950]. 
$E_{1 \mathrm{p}}=$ Änderung der Bandkante des Valenzbandes pro Einheitsdilatation,

$c_{\mathrm{ii}}=$ elastische Konstante für eine akustische Longitudinalwelle, in der jeweiligen Ausbreitungsrichtung,

$m_{\mathrm{p}}{ }^{*}=$ effektive Masse des Defektelektrons.

Die Formel ist unter der Voraussetzung sphärischer Energieflächen im Impulsraum abgeleitet und berücksichtigt nur das Vorhandensein einer Art von Defektelektronen. Bei Hinzunahme der leichten Löcher tritt eine Modifizierung von (4) ein, die für die folgenden Überlegungen außer Betracht bleiben kann, da die leichten Löcher wegen ihres kleinen Beitrages zur Leitfähigkeit das Beweglichkeitsverhalten unter Druck nicht wesentlich beeinflussen können. Die Deformationspotentialformel beruht auf der Wechselwirkung von akustischen Longitudinalwellen mit den Leitungselektronen. Die Untersuchungen von Sмгтн ${ }^{17}$ über den Piezo-Widerstandseffekt haben aber gezeigt, daß eine Scherung des Germaniumgitters in gewissen Kristallrichtungen den Widerstand merklich beeinflussen kann, so daß die Wirkung von Schubwellen auf die Beweglichkeit nicht vernachlässigt werden kann. Für n-Germanium wurde die Theorie von Duмке ${ }^{18}$ in dieser Richtung ergänzt. Nach Adams ${ }^{19}$ kann bei p-Germanium durch Scherbeanspruchung die Entartung an der Kante des Valenzbandes aufgehoben werden, was Anlaß zu einem zusätzlichen Streupotential gibt. Messungen der Schallgeschwindigkeit bei hohen Drucken ${ }^{20}$ haben jedoch gezeigt, daß die Schubwellengeschwindigkeit mit der Schwingungsrichtung [110] um fast eine Größenordnung weniger druckabhängig ist als die Longitudinalwellengeschwindigkeit, so daß mit einiger Berechtigung die Änderung des Einflusses der Schubwellen bei Anwendung hoher Drucke unberücksichtigt bleiben kann. Ein möglicher Einwand gegen die Deformationspotentialtheorie ist, daß sie die $T^{-2,33}$-Temperaturabhängigkeit der Löcherbeweglichkeit nicht korrekt wiedergibt. Da bis heute keine befriedigende Erklärung für die Abweichung gefunden wurde und über den zugrunde liegenden Mechanismus nichts bekannt ist, kann auch über dessen etwaige Druckabhängigkeit nichts ausgesagt werden.

Wenn man von den oben gemachten Einschränkungen absieht, kann man die Zunahme der Löcherbeweglichkeit unter Druck zurückführen auf Ände-

17 Сн. S. Sмітн, Phys. Rev. 94, 43 [1954].

18 W. P. Duмke, Phys. Rev. 101, 531 [1956].

19 E. N. Adams, Phys. Rev. 96, 803 [1954]. rungen der elastischen Konstanten $c_{\mathrm{ii}}$ des Deformationspotentials $E_{1 \mathrm{p}}$ und der effektien Masse $m_{\mathrm{p}}{ }^{*}$. Die Druckabhängigkeit einer dieser Größen ist bekannt. Messungen der elastischen Konstanten $c_{11}, c_{12}$ und $c_{44}$ bei Drucken bis $12000 \mathrm{kp} / \mathrm{cm}^{2}$, abgeleitet aus Schallgeschwindigkeitsmessungen, ergaben eine lineare Zunahme dieser Konstanten mit dem Druck in Übereinstimmung mit Resultaten von $\mathrm{MCS}_{\mathrm{CImiN}}{ }^{21}$, dessen Druckbereich $3500 \mathrm{kp} / \mathrm{cm}^{2}$ beträgt. Für die elastischen Konstanten $c_{\mathrm{ii}}$ bei Ausbreitung der Longitudinalwellen in [100], [110] und [111] ergab sich übereinstimmend für die drei Richtungen eine $\mathrm{Zu}$ nahme von $c_{\mathrm{ii}}$ um $3,9 \%$ bei $10000 \mathrm{kp} / \mathrm{cm}^{2}$, so $\mathrm{da} \beta$ eine Mittelung über die verschiedenen Richtungen des Wellenvektors sich erübrigt.

Die Änderung des Deformationspotentials mit dem Druck ist der Messung nicht direkt zugänglich. Aus dem Druckkoeffizienten der verbotenen Zone und dessen Druckabhängigkeit kann man nicht auf die Änderung von $E_{l \mathrm{p}}$ schließen, da sich in diesem Fall die Einflüsse von Valenz- und Leitfähigkeitsband überlagern. Mangels genauer Kenntnis der Änderung des Deformationspotentials wird deshalb die Annahme gemacht, daß $E_{1 \mathrm{p}}$ druckunabhängig ist. Um die beobachtete Beweglichkeitszunahme von $1,35 \%$ bei $10000 \mathrm{kp} / \mathrm{cm}^{2}$ durch Änderungen der effektiven Masse $m_{\mathrm{p}}{ }^{*}$ der schweren Löcher zu deuten, muß man eine Zunahme von $m_{\mathrm{p}}{ }^{*}$ von $1 \%$ bei $10000 \mathrm{kp} / \mathrm{cm}^{2}$ annehmen. Wegen der $m_{\mathrm{p}}{ }^{*-5 / 2}-\mathrm{Ab}$ hängigkeit der Beweglichkeit ergibt sich mit der 3,9-proz. Zunahme der elastischen Konstanten $c_{\mathrm{ii}}$ der beobachtete Verlauf der Löcherbeweglichkeit.

Hinsichtlich der Übereinstimmung von Elektronendriftbeweglichkeit in p-Germanium und Leitfähigkeitsbeweglichkeit in n-Germanium in Abhängigkeit vom Druck gilt analog das bei der Löcherbeweglichkeit Gesagte. Da die Abnahme der Elektronenbeweglichkeit mit wachsendem Druck wesentlich stärker ist als die Zunahme der Löcherbeweglichkeit, ist die Übereinstimmung des direkten und des indirekten Verfahrens noch überzeugender. Der zu geringe Absolutwert bei der Elektronendriftbeweglichkeit dürfte nicht allzu schwerwiegend sein, da man Grund zu der Annahme hat, daß jeder zusätzliche Streumechanismus, der Ursache für die zu geringe Beweglichkeit sein könnte, wesentlich schwächer druckabhängig ist als derjenige, der für die beobachtete Beweglichkeits-

\footnotetext{
20 J. Koppelmann u. G. Landwehr, Veröffentlichung in Vorbereitung.

${ }^{21}$ H. J. McScrmin, J. Acoust. Soc. Amer. 30, 314 [1958].
} 
abnahme verantwortlich ist. Für die Druckabhängigkeit der Elektronenbeweglichkeit liegt inzwischen eine detaillierte Theorie vor ${ }^{15}$, die auf Vorstellungen von PaUl und Brooks ${ }^{14}$ sowie Herman ${ }^{22}$ beruht. Diese Autoren haben als erste unabhängig voneinander vorgeschlagen, den Abfall der Elektronenbeweglichkeit folgendermaßen zu deuten: Das [111]-Energieminimum im $k$-Raum, das normalerweise bei $\mathrm{n}$ Germanium mit Elektronen besetzt ist, entfernt sich mit zunehmendem Druck vom Valenzband, während das [100]-Minimum, das bei $p=0$ ca. $0,2 \mathrm{eV}$ über dem [111]-Minimum liegt, seine Lage kaum ändert. Dadurch entleert sich mit wachsendem Druck das [111]-Energieminimum auf Kosten des in [100] gelegenen, und es tritt eine zusätzliche, als Zwischenbandstreuung (interband-scattering) bezeichnete Erscheinung auf, die zu der raschen Widerstandsabnahme führt. $\mathrm{Zu}$ der durch Zwischenbandstreuung hervorgerufenen Beweglichkeitsänderung tritt diejenige, die sich durch die Deformationspotentialformel abschätzen läßt und auf Änderungen der elastischen Konstanten, der longitudinalen und transversalen effektiven Masse der Elektronen sowie des Deformationspotentials beruht. Die Beweglichkeitsänderung auf Grund dieser Faktoren ist vor allem bei relativ niedrigen Drucken wirksam, während bei höheren Drucken die Streuung zwischen den Minima dominiert.

Es wurde vor kurzem festgestellt, daß zu gleicher Zeit mit den Untersuchungen, von denen hier berichtet wird, eine unabhängige Messung der Druckabhängigkeit der Driftbeweglichkeit von Elektronen in pGermanium bei Drucken bis zu $30000 \mathrm{kp} / \mathrm{cm}^{2}$ bei
Zimmertemperatur mit der Methode von Lawrence und Gibson durchgeführt wurde ${ }^{23}$. Die Löcherdriftbeweglichkeit wurde von SмIтH bei Drucken bis $12000 \mathrm{kp} / \mathrm{cm}^{2}$ bei den Temperaturen 296 und $195^{\circ} \mathrm{K}$ gemessen. Weiter wurden bei verschiedenen Temperaturen Widerstandsmessungen unter Druck an p-Germanium ausgeführt. Die Ergebnisse von Sмiтh sind mit den hier mitgeteilten in guter Übereinstimmung. Die Zunahme der Leitfähigkeitsbeweglichkeit von Löchern ${ }^{23}$ lag zwischen $(1,2 \pm 0,1) \%$ und $(1,38 \pm 0,06) \%$ bei $10000 \mathrm{kp} / \mathrm{cm}^{2}$, für die $\mathrm{Zu}$ nahme der Driftbeweglichkeit bei $296^{\circ} \mathrm{K}$ wurde der Wert $(1,1 \pm 1,1) \%$ gefunden. Auch bei den Messungen der Elektronendriftbeweglichkeit unter Druck besteht innerhalb der Fehlergrenze von $\pm 2 \%$ gute Übereinstimmung.

Den Herren Prof. Dr. R. Vieweg und Prof. Dr. H. Moser danke ich für die ständige Förderung der Arbeit, Herrn Prof. Dr. M. Kohler für wertvolle Diskussionen. Den Herren Dr. E. Arends, AEG/Gleichrichterfabrik Belecke, und Dipl.-Ing. F. W. Dehmelt, Telefunken/ Röhrenwerk Ulm, danke ich für die freundliche Überlassung von Germaniumkristallen. Herr Dr. W. FLECHSIG stellte mir die Einrichtungen des Halbleiterlaboratoriums der Physikalisch-Technischen Bundesanstalt zur Verfügung, Herr Dr. J. Gielessen die Hochdruckapparatur. Herrn H. J. Engelmann bin ich für die Herstellung der Versuchskristalle und technische Assistenz zu Dank verpflichtet. Der benutzte Doppelimpulsgenerator war eine Leihgabe der Deutschen Forschungs gemeinschaft, für dessen Überlassung gedankt wird.

22 F. Herman, Phys. Rev. 95, 847 [1954].

23 A. C. Sмiтн, Technical Report No. HP2 (1958), Gordon McKay Laboratory, Harvard University.

\title{
Zur Anomalie des Hall-Koeffizienten von schwad p-dotiertem InAs'
}

\author{
Von H. Rupprecht und H. Weiss \\ Aus dem Forschungslaboratorium der Siemens-Schuckertwerke AG, Erlangen \\ (Z. Naturforschg. 14 a, 531-535 [1959]; eingegangen am 24. März 1959) \\ Herrn Prof. Dr. E. Mollwo zum 50. Geburtstag
}

\begin{abstract}
Durch Potentialmessungen längs der HaLL-Strecke zwischen den beiden HaLl-Sonden läßt sich zeigen, daß der anomale Temperaturverlauf der HALL-Spannung an schwach p-leitendem InAs nach Sandstrahlen von einer dünnen n-leitenden Oberflächenschicht herrührt. Es lassen sich aus den Meßergebnissen einige Abschätzungen über den $\mathrm{H}_{\mathrm{ALL}}-\mathrm{Koeffizienten} \mathrm{der} \mathrm{n-leitenden} \mathrm{Schicht} \mathrm{und} \mathrm{das}$ Beweglichkeitsverhältnis von Löchern und Elektronen vornehmen.
\end{abstract}

In einer früheren Arbeit ${ }^{1}$ ist gezeigt worden, daß die elektrischen Eigenschaften von schwach p-dotierten Einkristallen aus InAs entscheidend von der Oberflächenbehandlung der Proben abhängen: Sand- strahlen oder Schmirgeln bewirkt anomalen Temperaturverlauf des HALL-Koeffizienten, d. h. zweimaligen Vorzeichenwechsel beim Abkühlen unter Zim1 H. Rupprecht, Z. Naturforschg. 13 a, 1094 [1958]. 